\title{
Measuring Software Architecture Stability Evolution in Object-oriented Open Source Systems
}

Hassan Almousa, Mamdouh Alenezi

College of Computer \& Information Sciences

Prince Sultan University

Riyadh 11586, Saudi Arabia

hassan.almousa@outlook.com

malenezi@psu.edu.sa

ABSTRACT: Stability is the capability of a software artifact to stay intact while adapting to new changes and requirements. Software architecture demonstrates the structure of a software system which can be presented as software components and their inter connections. Measuring the stability of software architecture is an important endeavor that can help developers or project managers to make them aware about the situation of the software being developed. Many software metrics have been introduced to measure the stability of the software architecture. Some of these metrics measure software architecture at package-level while other ones measure it at class-level. The principal goal of this paper is to come up with a new easy mechanism to measure the stability evolution in open source Java systems. Five different systems with ten versions are analyzed with respect to the new suggested mechanism of measuring stability evolution.

Keywords: Software Architecture, Maintainability, Stability, Software Metric, Measurement

Received: 12 December 2017, Revised 23 January 2018, Accepted 31 January 2018

DOI: $10.6025 /$ jitr/2018/9/2/35-47

(C) 2018 DLINE. All Rights Reserved

\section{Introduction}

Measurement in software engineering is a central feature to assess software quality characteristics such as maintainability, reliability, and portability. Software measurement [25] is not only about assessing software products but it can be used to assess the software development process. These measurements can contribute heavily in understanding software and processes in many ways for example by using software metrics, project managers will be able to know about the software status and evaluate the quality of various artifacts produced throughout software development. Software metrics are needed to measure various 
software attributes at different phases of the software development. Software metrics can be helpful in effectively measure different stages of the software development life cycle. Continuously measure software characteristic and development process usually supply timely management useful information about improving both process product. In this paper, a new mechanism will be designed to evaluate a sub quality characteristic which is the software stability, and then a case study will be discussed to see how the new mechanism will evaluate a set of systems. The remainder of this paper is organized as follows. Software architecture is discussed in Section 1. Software stability is defined in Sections 2. Our new stability metric is introduced in Section 3. A case study to examine the new stability metric is presented in Section 4. Finally, this study is calculated in Section 5.

\section{Software Architecture}

Software structure is usually designed right after collecting and studying the software requirements. Throughout this stage the software is built with regards of components and interconnections that tie these components with each other [1]. This structure will demonstrate the architecture for specific software. Moreover, the software system architecture is the initial design piece that reports quality objective such as maintainability, stability, modifiability, and performance [13]. Software architecture divides the system structure with regards of components and their connections from the detailed design of these individual components. Software architecture can be defined at two levels, high level and lower level. At high level, software is decomposed into subsystem where at lower level, a software is decomposed into modules and components. Interfaces are used to connect subsystems or components with each other [21]. Software architecture signifies the critical design decisions that are toughest to change and decide the overall system proprieties [2]. These decisions should be before working on the detailed aspects of the system. These architecture decisions not only made at the component level, but they usually include the overall system components and constrains. After deciding about all architecture decisions, the team can work in designing and delivering individual components [10].

Software architecture is not just how software is constructed, but it guides the software evolution [10]. Now days it is impossible to have a software product remains as it was since it is being developed. The software will evolve continuously, requiring continuous development and maintenance [9]. Changes come throughout software's life in term of adding new features, modifying existing features, removing some features or fixing software defects. All these actions that may accrue on the software architecture can be referred as signs of aging. Signs of aging can negatively affect the software architecture and make it deviated from the original architecture. This deviation could harm the software architecture and make it complex and hard to maintain. By observing a software evolution, information from the software architecture will be obtained by evaluating this architecture. Therefore, software metrics are needed to evaluate and assess to avoid the negative impacts of signs of aging. Quantitative information that obtained from the software metrics can help developers to build systems with better quality.

\section{Stability}

The main goal of evaluating software architecture is to validate the software architecture using systematic procedures [13]. This evaluation goal is to make sure that the architecture under examination fulfil one or more of software quality characteristics. According to ISO/IC square quality standards, quality characteristics consist of four characteristics: functionality, usability, reliability, efficiency, portability, and maintainability [22]. Maintainability is defined as the capability of a software product to be modified. Maintainability is ether categorized into a set of sub characteristics: stability, analyzability, changeability, and testability. At the architecture level, these characteristics will be refined into coupling and modularity to be able to be evaluated and assessed[15]. As mentioned before stability is a sub characteristic under maintainability and it is defined as the capacity of the software product to stay unaffected when facing new requirements and/or changing in the environment. Stability evolution is measured by computing the differences between the stability of two versions while the software is evolving. On other hand, it is impossible to keep a software product unchanged. Then, what the software is supposed to do? The answer is these new changes have to be accommodated in the software architecture, and if not these changes will lead to the degradation of the usefulness of the software product [2]. From what have been said before, it noticeable that stability is a primary criterion for evaluating an architecture which can be seen as an indicator for software maturity [9] [14]. If a software product has some components and these components are unstable, the software architecture will require high maintenance cost and effort [19].On other hand, once an architecture for a software component is recognized as a stabile component, this component can be considered as a reusable component.

\section{Literature Review}

$36 \quad$ Journal of Information Technology Review Volume 9 Number 2 May 2018


This section browses different attempts in the literature to measure the stability of specific software. Aversano et al. [3] evaluated the software architecture for a set of open source software projects. Stability is the characteristic that is examined in order to evaluate the software core architecture. The evolution of certain software is considered when the software components are changed during the software releases. Two metrics were proposed to measure the stability of each release, Core Design Instability (CDI) and Core Call Instability (CCI). Both metrics provided a measure of how much the architecture of a software system changed passing from a release to another one. CDI metric finds the change in terms of number of packages and CCI finds the change in terms of number of the interactions among packages. Smaller values mean less change which means greater stability. All these metrics are based on calculating fan-in and self-call for software packages.

Alshayeb et al. [4] mentioned that none of the existing measures have included all class aspects such as class relationships, attributes, and methods. First, they identified all properties that affect the class stability; these properties are class access level, class interface level, inherited class name, class variable, class variable access-level, method signature, method access level, method body. Then from these properties, they proposed Class Stability Metric (CSM). Stability is calculated by counting the number of unchanged properties between two classes in version $i+1$ and version $i$ divided by the maximum possible change value, then the summation of all these properties is divided by the number of the properties which is eight. The result their empirical study indicated that their metric is highly negatively correlated with maintenance effort.

Li et al [5] proposed new metrics to measure the stability of software designs. Authors in [5] highlighted that metrics that are discovered by Chidambe \& Kemerer [8] can't measure change in the class name, class number, and class inheritance relations. Tackling this deficiency of C\&K metrics, they proposed three new metrics: System Design Instability (SDI), Class Implementation Instability (CII), and System Implementation Instability (SII). The main purpose was to justify how the information that is gathered from theses metrics can help project managers to modify software project plan. Their experimental analysis showed that SDI and CII can measure Object Oriented aspects that are different from the aspects that are measured by C\&K metrics.

Abdeen et al. [6] proposed a number of coupling and cohesion metrics that evaluate packages modularization in legacy objectoriented systems. These metrics are Index of Inter-Package Usage (IIPU), Index of Inter-Package Extending (IIPE), Package Focus (PF), Index of Package Service Cohesion (IPSC), and Index of Package Changing Impact (IPCI). They defined these metrics with respect to some modularity principles that are related to packages. Examples of these principles are information hiding, changeability and communality of goal. These metrics are defined with respect to inter-class dependencies: method call and inheritance relationships. They validated these against the mathematical properties that have to be existed in any cohesion or coupling metric.

Jazayeri et al. [10] did retrospective analysis to assess the architecture stability for twenty releases of telecommunication software. The evaluation was intended to help project managers to predict how the future of the architecture will look like. They used simple metrics, module size, number of modules changed, number of modules added, number of modules changed in the same sequence of release, number of programs in the same version of release, to observe the effect of evolution on pair of releases.

Abreu and Melo. [11] evaluated the impact of object oriented design on software quality characteristics such as defect density, failure density, and normalized rework. They used a set of object oriented design metrics called MOOD. These metrics are method hiding factor (MHF), attribute hiding factor (AHF), method inheritance factor (MIF), attribute inheritance factor (AIF), polymorphism factor (POC) and coupling factor (COF). To quantify the impact of $\mathrm{OO}$ design on software quality, they developed a predictive model. The results show that the design alternatives may have a strong influence on resulting quality.

Olague et al. [12] utilized entropy to reduce spikes in the original SDI metric that is produced by Li.et al [5] and proposed the new SDIe metric. They discussed the dynamic nature of the agile development process that could obscure an analysis of software stability. Furthermore, this study noted that the SDIe metric is easier than the original SDI metric in computing the stability. The justification is the fact that SDIe is able to be automated instead of requiring the close investigation of code by human judgment. The SDIe metric is calculated using the number classes added, deleted, changed and unchanged from the previous versions. SDIe metric is theoretically investigated and validated using the Kitchenham criteria [24] and the Zuse requirements [23] for software measures. Moreover SDIe is empirically tested over two software projects by comparing SDIe metric with the original SDI, using SDIe to assess the software evolution, and comparing SDIe metric to the Chidamber and Kemerer [8].

Tonu et al. [13] proposed an approach that evaluates stability for a particular software architecture. The approach is based on Journal of Information Technology Review Volume 9 Number 2 May 2018 
analyzing the changes in the software's aspects form one release to another. Software aspects can be structural, behavior, or economical, their focus was on the structural aspects only. Growth rate, changes rate, coupling, cohesion are the measures that are applied in their retrospective analysis. Then, evolution sensitivity and evolution critical parts are identified by observing how the subsystems are interconnected. This approach is empirically evaluated on two spreadsheet applications by selecting nine releases for each application.

Ratiu et al. [17] started by defining two threshold measurements that are used to identify which structure is considered a god class or data class. First measurement is used to identify god classes and it is based on these metrics: Access to Foreign Data (ATFD) and Weighted Method Count (WMC), Tight Class Cohesion (TCC),Number of Attributes (NOA). While the another measurement is used to identify data classes and it is based on these metrics: Weight of a Class (WOC), Number of Methods (NOM), Weighted Method Count (WMC), Number of Public Attributes (NOPA) and Number of Accessory Methods (NOAM). Then, they proposed two measurements that are applied on the history of a design structure. One of these measurements is used to measure the stability of a class ( $\mathrm{Stab}$ ) and the other one is used to measure the persistence of a design flaw (Pers). A class is considered stable with respect to measurement $M$ version $i$ and number of versions if there is no change in the measurement $M$. Their approach is applied on three case studies: two in-house projects, and one on a large open source framework. By observing the data while applying their approach, they discussed whither classes are god classes or data classes.

Bansiya et al. [18] introduced a framework to evaluate architecture stability that is based on quantitative assessment on the changes in versions using object oriented metrics. The framework consists of four steps to calculate the extent-of-change measure. First step is identifying structure characteristics that evaluate the architecture of framework. There are two types of structure characteristics: static and dynamic. Example of static structure characteristics are number of classes, number of class hierarchies, number of single and multiple inheritances, and average depth and width of class inheritance hierarchies. Examples of dynamic structure characteristics are number of services a class provides, class coupling, and number of inheritance related classes. Second step is defining metrics for each one of these structure characteristics. Third step is collecting the data from the defined metrics by applying theme on a case study. Finally, for each release the extent-of-change is calculated by normalizing the values of these metrics. Once all values are normalized, the aggregate-change is calculated by summation of these values. Then the extent-of-change is calculated by taking the difference of the aggregate change value of a version $i$ with the aggregate change value of the first version. The extent of change measure can be used as an indicator to identify the stability for a particular system structure, low number indicates high stability.

\section{Designing Stability Metric}

Object oriented design concepts are very important factors that contribute in the software development. The reason behind that is these concepts address fundamental concerns about software adaption and evolution. Information hiding is one of the object oriented concepts and it is used to decide which information should be visible and which information should be hidden. Inheritance is another concept of object oriented that is used for sharing and reusing properties between classes. Concurrent processing is another concept of object oriented and it describes how software's objects will interact with each other by invoking classes methods [21]. These concepts can be evaluated for particular software in order to recognize the software architecture. In this paper, the focus will be on the concepts that affect the software architecture in term of concurrent processing. It has been recognize from the literature review that have been reviewed in the previous section that most infusing attributes which are used in order to determine the stability for the software architecture are size, coupling and cohesion. Some researches use coupling and cohesion at the class level while other use coupling and cohesion at architecture level by considering the software packages. Furthermore, most of object oriented metrics that measure the software architecture for particular system are class-level metrics [4] [8] [17]. In this paper we are aiming to introduce a metric that is used to measure the stability of the software architecture based on these three attributes: size, coupling and cohesion.

\subsection{Identifying Software Architecture Attributes}

Software architecture attributes that will be used to measure the stability for the software architecture will be discussed in this section. Each one of these attributes (Size, Cohesion and Coupling)nominated to contribute in designing the new stability metric. Size provides high view about the amount of functionalities that have been developed for a particular system during its life cycle. These functionalities will be represented at the end for each class in the system either by adding new line of codes or methods or imported packages or adding new interfaces. Therefore, size will be used as a good indicator to know if new functionalities have been added in a system. This indicator can be used as a measure to recognize the stability for a particular system and many researchers used this measure to study the stability in their studies like [12], [14] and [18]. Coupling is the

\begin{tabular}{llllll}
\hline 38 & Journal of Information Technology Review Volume 9 & Number 2 May 2018 \\
\hline
\end{tabular}


degree that can be pointed out in order to know how a class has dependences among other classes within the software architecture. In a brief way, coupling illustrates the relationships of a class with other classes. There is a contradiction between coupling and software engineering factors like maintainability, testability and fault-proneness. Let's consider the case of maintainability to present how coupling will be accrued. If a developer edits the software architecture by making a modification in a class, this change may require a modification on one of classes that connect with this class. It is clear from the above that coupling can be used as good indicator to recognize the change in the software architecture by observing coupling between the software versions. This reorganization will tack place by measuring the coupling for a class during the software life cycle. There are many metrics have been introduced to measure the coupling at class-level. These metrics are Coupling between Objects, Message Passing Coupling, Efferent Coupling, Afferent Coupling, Response for Class, and Local Methods Calls. Many researches used coupling as a measure in their study like [5] [6], [11], [13], [14] and [19]. Cohesion is used to identify the degree of how class's elements are related to each other. In another way class cohesion describes the relationships between the class attributes and methods. A class will be pointed out as strong cohesion class, if there is a strong overlap between its attributes and its methods. Moreover, designing strong class cohesion is seen as a good quality attribute when evaluating the software architecture. On the other hand, there is a contradiction between designing high cohesive and complex class. Because designing very high cohesive class will lead this class to some degree of complexity. The optimal way to avoid this kind of concern is having threshold for cohesion while designing the software architecture. The previous sentences were giving overview about the class cohesion and it benefits and drawback and it is clear that cohesion can be used as an attribute to recognize the stability for the software architecture when a version of system compare with other system versions. Many researchers used cohesion as a measure in their study like [5], [12], [13] and [14].

\subsection{Selecting the Software Metrics for Size, Cohesion and Coupling Attributes}

Software quality characteristics can't be evaluated directly. They have to be refined into sub-characteristics. These subcharacteristics will be refined into attributes. The refinement attributes will be measured by using some metrics. In our case, the refinement attributes are size, coupling and cohesion. In this section, the software metrics for each one of refinement attributes that have been discussed previously will be identified and defined. In order to identify the metrics that will be nominated to be helped in designing the new metric, we used a software metrics tool to identify all metrics that give measurement at the classlevel. Only metrics that measure class-level with concerning size, coupling and cohesion are selected. Table 1 groups the metrics by the attributes and each metric in these groups is defined. However, not all metrics that are identified in table 1 will be selected to design the new metrics. The reason behind that is the attention will be given only to metrics that their results are not correlated when they are calculated for software. If there are two metrics their results are strongly correlated, only one of them will be selected. Spearman correlation analysis will be used to identify the metrics that are strongly correlated in each group (size, cohesion and coupling) [26].

\subsection{Designing the Stability Metric}

Stability is the ability of a class to remain unchanged when new requirements are introduced in a system. On the other hand, preventing a system from adding new features something is hardly to achieve. The system has to accommodate any new requirement to get the end users' satisfaction. If this not happened, the end user's will look to the systems competitors. The stability of a system can be measured by observing the system evolution. In our case the attributes that have been selected will be observed. This observation will take place by recognizing the difference between the systems' versions [15]. One of the measurements proposed by Ratiu et al in [17] is the stability of a class (Stab). They mentioned a class $A$ is considered stable with respect to measurement $M$ version $i$ and number of versions $N$ if there is no change in the measurement $M$ as illustrated in equation (1) and equation (2).

$$
\begin{aligned}
& \operatorname{stab}_{i}(C, M)=1, M_{i}(\mathrm{C})-M_{i-1}(C)=0 \\
& \operatorname{stab}_{i}(C, M)=0, M_{i}(\mathrm{C})-M_{i-1}(C) \neq 0
\end{aligned}
$$

Once the stability of a class is identified in version $i$, the stability of this class in version $N$ is calculated as the number of versions in which a class was changed over the total number of versions. Equation (3) describes how the stability will be calculated

$$
\operatorname{stab}_{1 \ldots . . n}=\frac{\sum_{i=1}^{n} \operatorname{stab}_{i}(C, M)}{n-1}
$$




\begin{tabular}{|c|c|c|}
\hline Group & Metric & Detention \\
\hline \multirow{4}{*}{ Size } & NLOC & Counts number of lines of Code in a class \\
\hline & PACK & Counts number of packages that are imported in a class \\
\hline & INTER & Counts number of Interfaces that are implemented in a class. \\
\hline & No. methods & Counts number of methods that are defined in a class \\
\hline \multirow{3}{*}{ Cohesion } & LCOM 1 & $\begin{array}{l}\text { Lack of Cohesion of Methods and it shows how the methods of a class are } \\
\text { not related to each other to achieve the aims of the class. }\end{array}$ \\
\hline & LCOM 2 & $\begin{array}{l}\text { Lack of Cohesion of Methods and it shows how the methods of a class are } \\
\text { not related to each other to achieve the aims of the class. }\end{array}$ \\
\hline & $\mathrm{COH}$ & $\begin{array}{l}\text { Cohesion and it shows how well the methods of a class co-operate to achieve } \\
\text { the aims of the class. }\end{array}$ \\
\hline \multirow{6}{*}{ Coupling } & $\mathrm{CBO}$ & $\begin{array}{l}\text { Coupling between objects and it counts the number of classes that are } \\
\text { coupled to a particular class. }\end{array}$ \\
\hline & FAN-IN & $\begin{array}{l}\text { Afferent coupling and it counts the number of other classes that reference a } \\
\text { class }\end{array}$ \\
\hline & FAN-OUT & $\begin{array}{l}\text { Efferent coupling and it counts the number of other classes that referenced } \\
\text { by a class. }\end{array}$ \\
\hline & $\mathrm{RFC}$ & $\begin{array}{l}\text { Response for class and it is defined as a set of methods that can be executec } \\
\text { in response to a message received by another class. }\end{array}$ \\
\hline & MPC & $\begin{array}{l}\text { Message Passing Coupling and it counts the numbers of messages passing } \\
\text { among objects of a class. }\end{array}$ \\
\hline & LMC & Number of Local Method called in a class. \\
\hline
\end{tabular}

Table 1. Class - level metrics

The above equations measure the stability at class-level but in this study the concern is paid to measure the stability at architecture-level. Moreover, the above equations identify the stability based on one method, but in this study there is three attributes (Size, Cohesion and Coupling) and each one of these attributes has a set of metrics. In this study, Equation (1), equation (3) will be modified to measure the stability for the software architecture for a system. Instead of calculating the stability for a system by considering each class in the system and using only a single metric, the stability for the whole system will be measured by considering more than one metrics. The following steps show how the stability for the software architecture will be measured.

Step 1: Extracting class-level metric using software metrics tool. The value of the class-level metrics for the selected attributes will be calculated by extracted their data using a software metrics tool for each class in the system.

Step 2: Calculating the average for the class-level metrics. The average will be calculated for each metric in the system after the metrics values being extracted.

Step 3: Calculating the aggregated metric. The average of all metrics will be aggregated in one metric to be used to compare its 
value with the system's versions. Equation (4) describes how all metrics $M$ in version $i$ will be combined.

$$
\text { aggregated metric }_{i}=\sum_{i=1}^{j=n} A V G(M)_{i}
$$

Step 4: Do comparison between the system versions. Step 1, step 2 and step 3 will be repeated for each version $i$ for the selected system to obtained the aggregated metric. Each version $i$ will be considered stable with respect to the aggregated metric version $i$ and number of versions $N$ if there is no change in the aggregated metric. Metric (5) and metric (6) illustrate how the aggregated metric will be compared between the system versions.

$$
\begin{aligned}
& \text { stab }_{i}=1, \text { Aggregated Metric }_{i}-\text { Aggregated Metric }_{i-1}=0 \\
& \text { stab }_{i}=0, \text { Aggregated Metric }_{i}-\text { Aggregated Metric }_{i-1} \neq 0
\end{aligned}
$$

Step 5: Measuring the stability for the selected system. The stability for the selected system will be measured by evaluating the $s t a b i$, which is described in step 4, between a pair of versions for $N$ versions. After the stab evaluated for each version $i$, stab $i$ will be aggregated for all versions $N$ then it will be divided by total number of versions $N-1$. Equation (7) illustrates how the system stability will be measured.

$$
\text { System }_{\text {stab }}{ }_{1 \ldots . . n}=\frac{\sum_{i=2}^{n}{ }_{i=n} t a b_{i}}{n-1}
$$

\section{Case Study}

In this section, a case study will be presented in which five object-oriented java systems are selected. All these systems are open source systems and they downloaded from Source forge library. Since our stability metric at its early stage requires at least a pair of versions, it is mandatory to have a large number of versions to be trained. From this concern we agreed to download ten versions for each one of the selected systems. One of the crucial requirements to start applying this study is to choose a software metric tool to extract the results of class-level metrics. At the first of this section we will talk about the benefits of open source systems. Then, the selected software metric tool will be discussed. After that, a summary about the selected systems will be given. Finally, the results of applying our stability metric will be shown.

\subsection{Open Source Systems}

Open source systems have received a lot of attention to be used by practitioners in these days. The reason behind that is the availability of a large number of systems in different domains. A researcher downloads an open source system for applying the required analysis form his or her study. A programmer modifies an open source system to develop a particular need. It is obvious from the above that the availability of open source system will reduce cost, effort, and time to obtain a system meets a desired need [27]. There are many open source libraries are available on the internet. These libraries are sourceforge, Gethub, Google code and Tigers. In this study source forge will be used to download five systems with ten versions. In order to evaluate our stability metric, we realized that it is necessary to put some criteria to help us in selecting an open source system. Following are the criteria that we stick on to select a set of open source systems:

- All selected systems have to be written in Java.

- All selected systems have to be object-oriented.

- The selected systems have to be from different domains.

- There are multiple versions for the selected systems.

- There is a variation in the number of classes for the selected systems.

Table 2 gives a brief summary for the selected open source systems.

\subsection{Software Metric Tool}

One of the fundamental requirements for out stability metric is to have a tool provides many metrics at class -level for the attributes that are selected to design our stability metric. Another requirement is the tool should be able to analysis systems that 


\begin{tabular}{|l|l|l|}
\hline System & \multicolumn{1}{|c|}{ Domain } & Description \\
\hline SQSim & Scientific / Engineering & $\begin{array}{l}\text { This system used to model simple probabilistic queue, } \\
\text { dynamic, factories, business, services and other }\end{array}$ \\
\hline Bar Code Generator & Development / Dynamic content & $\begin{array}{l}\text { This system used to generate barcode of different types } \\
\text { like EANB, UPCA etc. }\end{array}$ \\
\hline Ezbilling & Finance & $\begin{array}{l}\text { This system used to manage the invoices that will be } \\
\text { issued. }\end{array}$ \\
\hline Catan & Game & $\begin{array}{l}\text { This system allows anyone to play board game against } \\
\text { the computer. }\end{array}$ \\
\hline EuroBudget & Accounting & This system is a free personal accounting tool. \\
\hline
\end{tabular}

Table 2. Selected open source system

are written in Java. During our search for the most appropriate tools that accommodate our needs, we found many software metric tools. Some of these tools provide many metrics for the selected attributes while other provides few metrics. Examples of these tools are JHWAK, Source Code Metric Plugin and Simple Code Metric Plugin. In this study we used JHAWK tool to extract the results of class-level metrics. JHAWK is a standalone application provides plenty of metrics at different levels like package-level, class-level and method-level. One of the most important features that attract us to select JHAWK is the ability to choose only the metrics that we want to be extracted when analyzing a system. These metrics can be managed through preferences in tool setting. JHAWK has the ability to extract the results of the metrics in different formats like CSV, HTML and XML.

\subsection{Appling the Stability Metric}

Our stability metric needs to be validated by selecting a set of open source systems. The main objective from this validation is to see how our stability metric will evaluate the selected systems. As mentioned previously not all metrics that contribute to measure the size, cohesion and coupling will be nominated to design our stability metric. Therefore, spearman correlation analysis is applied to discard the metrics that are strongly correlated. All size, cohesion and coupling metrics' results for all systems' versions are extracted. Then, the related metrics are grouped with each other. For examples, coupling metrics will be combined in one group. Table 3 shows how metrics and their results are grouped by size, cohesion and coupling attributes.

\begin{tabular}{|c|c|c|c|c|c|c|c|c|c|c|c|c|c|}
\hline \multirow{2}{*}{ Class Name } & \multicolumn{4}{|c|}{ Size } & \multicolumn{3}{|c|}{ Cohesion } & \multicolumn{6}{|c|}{ Coupling } \\
\hline & NLOC & No. Methods & INTR & PACK & LCOM & LCOM2 & $\mathrm{COH}$ & RFC & CBO & MPC & F-IN & FOUT & LMC \\
\hline BarSet & 50 & \begin{tabular}{|r|}
6 \\
\end{tabular} & 0 & 0 & 0.2 & $\begin{array}{r}0 \\
\end{array}$ & 0.33 & 6 & 24 & 0 & 24 & 0 & 1 \\
\hline BarcodeEncoder & 4 & 2 & 0 & 0 & 0 & 2 & 0 & 2 & 16 & 0 & 14 & 2 & 0 \\
\hline BarcodePainter & 3 & 1 & 0 & 2 & 0 & 1 & 0 & 1 & 8 & 0 & 7 & 1 & 0 \\
\hline BaselineTextPainter & 24 & 3 & 1 & 5 & 1 & 4 & 0.33 & 3 & 5 & 0 & 4 & 1 & 0 \\
\hline CircularPainter & 55 & 3 & 1 & 8 & 1 & 4 & 0.33 & 3 & 3 & 0 & 1 & 2 & 0 \\
\hline CodabarEncoder & 84 & 10 & 1 & 0 & 0.01 & 30 & 0.08 & 11 & 7 & 1 & 4 & 3 & 1 \\
\hline Code11Encoder & 59 & 6 & 1 & 0 & 0.1 & 14 & 0.17 & 7 & 6 & 1 & 3 & 3 & 1 \\
\hline Code128Encoder & 186 & 7 & 1 & 1 & 0.02 & 17 & 0.11 & 8 & 7 & 1 & 4 & 3 & 2 \\
\hline Code39Encoder & 100 & 7 & 1 & 0 & 0.12 & 17 & 0.14 & 8 & 8 & 1 & 5 & 3 & 2 \\
\hline Code39ExtEncoder & 45 & 6 & 0 & 0 & 0.4 & 14 & 0.17 & 7 & 7 & 1 & 3 & 4 & 1 \\
\hline Code93Encoder & 127 & 7 & 1 & 0 & 0.12 & 17 & 0.14 & 8 & 8 & 1 & 5 & 3 & 2 \\
\hline Code93ExtEncoder & 51 & 6 & 0 & 0 & 0.4 & 14 & 0.17 & 7 & 7 & 1 & 3 & 4 & 1 \\
\hline DirectGif89Frame & 29 & 3 & 0 & 3 & 0 & 0 & 1 & 3 & 3 & 0 & 2 & 1 & 0 \\
\hline EAN13Encoder & 46 & 4 & 0 & 0 & 0.33 & 8 & 0.25 & 5 & 8 & 1 & 5 & 3 & 0 \\
\hline EAN13TextPainter & 35 & 3 & 1 & 5 & 1 & 4 & 0.33 & 3 & 5 & 0 & 4 & 1 & 0 \\
\hline EAN8Encoder & 36 & 4 & 0 & 0 & 1 & 8 & 0.25 & 4 & 7 & 0 & 4 & 3 & 0 \\
\hline EAN8TextPainter & 34 & 3 & 1 & 5 & 1 & 4 & 0.33 & 3 & 5 & 0 & 4 & 1 & 0 \\
\hline \begin{tabular}{|l|} 
EANEncoder \\
\end{tabular} & 35 & 2 & 1 & 0 & 4 & 2 & 0 & 3 & 7 & 1 & 4 & 3 & 1 \\
\hline Gif89Encoder & 163 & 22 & 0 & 11 & 0.02 & 186 & 0.18 & 24 & 7 & 2 & 2 & 5 & 7 \\
\hline Gif89Frame & 67 & 12 & 0 & 3 & 0.04 & 68 & 0.13 & 13 & 6 & 1 & 4 & 2 & 2 \\
\hline GifColorTable & 94 & 11 & 0 & 0 & 0.06 & 40 & 0.25 & 11 & 5 & 0 & 1 & 4 & 3 \\
\hline GifPixelsEncoder & 240 & 12 & 0 & 0 & 0.14 & 66 & 0.17 & 12 & 1 & 0 & 1 & 0 & 10 \\
\hline HeightCodedPainter & 34 & 3 & 1 & 4 & 1 & 4 & 0.33 & 3 & 6 & 0 & 4 & 2 & 0 \\
\hline ImageUtil & 33 & 4 & 0 & 7 & 0 & 8 & 0.25 & 4 & 3 & 0 & 1 & 2 & 2 \\
\hline IndexGif89Frame & 10 & 2 & 0 & 0 & 0 & 0 & 0 & 2 & 3 & 0 & 2 & 1 & 0 \\
\hline Interleaved2of5Encoder & 38 & 4 & 0 & 0 & 0.11 & 8 & 0.25 & 5 & 7 & 1 & 4 & 3 & 0 \\
\hline InvalidAtributeException & 15 & 4 & 0 & 0 & 1 & 4 & 0 & 4 & 23 & 0 & 23 & 0 & 0 \\
\hline \begin{tabular}{|l|l} 
JBarcode \\
\end{tabular} & 121 & 23 & 0 & 9 & 0.03 & 244 & 0.12 & 23 & 9 & 0 & 4 & 5 & 4 \\
\hline
\end{tabular}

Table 3. Class-level metrics grouped by size, cohesion and coupling 
A spearman correlation analysis will be applied in each one of these groups. Table 4, table 5 and table 6 show the spearman correlation analysis results for size, cohesion and coupling attributes respectively.

\begin{tabular}{|l|c|c|c|c|}
\hline \multicolumn{5}{|c|}{ Size } \\
\hline & NLOC & No. Meth & INTR & PACK \\
\hline NLOC & 1 & & & \\
\hline No. Meth & 0.684145 & 1 & & \\
\hline INTR & 0.144081 & 0.309831 & 1 & \\
\hline PACK & -0.0677 & -0.12603 & -0.06122 & 1 \\
\hline
\end{tabular}

Table 4. Spearman correlation analysis results for size metrics

\begin{tabular}{|l|l|l|l|}
\hline \multicolumn{4}{|c|}{ Cohesion } \\
\hline & LCOM & LCOM2 & COH \\
\hline LCOM & 1 & & \\
\hline LCOM2 & -0.03591 & 1 & \\
\hline COH & 0.263737 & -0.03994 & 1 \\
\hline
\end{tabular}

Table 5. Spearman correlation analysis results for cohesion metrics

\begin{tabular}{|l|c|c|c|c|c|c|}
\hline \multicolumn{7}{|c|}{ Coupling } \\
\hline & RFC & CBO & MPC & F-IN & FOUT & LMC \\
\hline RFC & 1 & & & & & \\
\hline CBO & 0.712879 & 1 & & & & \\
\hline MPC & 0.46271 & 0.567566 & 1 & & & \\
\hline F-IN & 0.589835 & 0.869623 & 0.5455 & 1 & & \\
\hline FOUT & 0.577303 & 0.726984 & 0.333192 & 0.301873 & 1 & 1 \\
\hline LMC & 0.867593 & 0.655508 & 0.354002 & 0.530947 & 0.558953 & \\
\hline
\end{tabular}

Table 6. Spearman correlation analysis results for coupling metrics

When we see the results for spearman correlation analysis results for size group, we realized that there is a strong correlation between NLOC and number of methods. Therefore, only one of them will be chosen and in our case study we select NLOC. When we see the results for spearman correlation analysis results for cohesion group, we realized all metrics are not correlated with each 
other. Therefore, all of them are nominated to contribute in designing our stability metric. Finally, spearman correlation analysis results for coupling metric shows all coupling metrics are strongly correlated. Therefore, selection one of them is enough and we select coupling between objects (CBO). The reason for selecting this metric is $\mathrm{CBO}$ includes all dependences ingoing and outgoing [28].

Now size, cohesion and coupling metrics that will be nominated to design our stability metrics are identified. These metrics are NLOC, LCOM, LCOM 2, COH and CBO. We are ready now to apply the steps in following for each one of the selected systems to measure its architecture.

Step 1: Extracting class-level metric

Step 2: Calculating the average for the class-level metrics.

Step 3: Calculating the aggregated metric.

Step 4: Do comparison between the system versions.

Step 5: Measuring the stability for the selected system.

Table 7 shows the result for our stability metric after applying the previous steps on SQSim. Our stability metric evaluates the architecture for this system and it gives $76 \%$. We compare a pair of subsequence versions, and we realized the aggregated metric results for $V 2, V 6, V 7$ are different when they compare with $V 1, V 5$ and $V 6$.

\begin{tabular}{|l|l|l|l|l|l|l|l|l|l|l|l|}
\hline \multirow{4}{*}{ SQSim } & V1 & V2 & V3 & V4 & V5 & V6 & V7 & V8 & V9 & V10 & Stability \\
\cline { 2 - 11 } & 66.77478 & 66.75304 & 66.75304 & 66.75305 & 66.75304 & 66.84 & 66.75304 & 66.75304 & 66.75304 & 66.75304 & \multirow{2}{*}{$67 \%$} \\
\cline { 2 - 10 } & & 0 & 1 & 1 & 1 & 0 & 0 & 1 & 1 & 1 & \\
\hline
\end{tabular}

Table 7. Stability result for SQSim

Table 8 shows the result for our stability metric after applying the previous steps on Barcode Generator. Our stability metric evaluates the architecture for this system and it gives $89 \%$. We compare a pair of subsequence versions, and we realized the aggregated metric results for $V 2$ is different when it compared with $V 1$.

\begin{tabular}{|l|l|l|l|l|l|l|l|l|l|l|l|}
\hline \multirow{3}{*}{$\begin{array}{l}\text { Barcode } \\
\text { Generator }\end{array}$} & $\mathbf{V 1}$ & $\mathbf{V 2}$ & $\mathbf{V 3}$ & $\mathbf{V 4}$ & $\mathbf{V 5}$ & $\mathbf{V 6}$ & $\mathbf{V 7}$ & $\mathbf{V 8}$ & $\mathbf{V 9}$ & V10 & Stability \\
\cline { 2 - 11 } & 112.8988 & 112.8388 & 112.8388 & 112.8388 & 112.8388 & 112.8388 & 112.8388 & 112.8388 & 112.8388 & 112.8388 & \multirow{2}{*}{$89 \%$} \\
\hline
\end{tabular}

Table 8. Stability result for Barcode Generator

Table 9 shows the result for our stability metric after applying the previous steps on Catan. Our stability metric evaluates the architecture for this system and it gives $67 \%$. We compare a pair of subsequence versions, and we realized the aggregated metric results for $V 3, V 4, V 5$ are different when they compare with $V 2, V 3$ and $V 4$.

\begin{tabular}{|c|l|l|l|l|l|l|l|l|l|l|l|}
\hline \multirow{4}{*}{ Catan } & V1 & V2 & V3 & V4 & V5 & V6 & V7 & V8 & V9 & V10 & Stability \\
\cline { 2 - 11 } & 92.3574 & 90.57128 & 90.57128 & 90.56011 & 90.57128 & 90.57128 & 90.57128 & 90.57128 & 90.57128 & 90.57128 & \\
\cline { 2 - 10 } & & 0 & 1 & 0 & 0 & 1 & 1 & 1 & 1 & 1 & \\
\hline
\end{tabular}

Table 9. Stability result for Catan

Table 10 shows the result for our stability metric after applying the previous steps on EuroBudget. Our stability metric evaluates the architecture for this system and it gives $22 \%$. We compare a pair of subsequence versions, and we realized the aggregated metric results for $V 2, V 3, V 4, V 5, V 6, V 7$ and $V 10$ are different when they compare with $V 1, V 2, V 3, V 4, V 5, V 6$ and $V 9$. 


\begin{tabular}{|l|l|l|l|l|l|l|l|l|l|l|l|}
\hline \multirow{3}{*}{$\begin{array}{l}\text { Euro } \\
\text { Budget }\end{array}$} & V1 & V2 & V3 & V4 & V5 & V6 & V7 & V8 & V9 & V10 & Stability \\
\cline { 2 - 11 } & 92.0466 & 91.39917 & 91.29543 & 93.62827 & 88.73376 & 88.72273 & 88.70638 & 88.70638 & 88.70638 & 88.72273 & \multirow{2}{*}{$22 \%$} \\
\cline { 2 - 11 } & & 0 & 0 & 0 & 0 & 0 & 0 & 1 & 1 & 0 & \\
\hline
\end{tabular}

Table 10. Stability result for EuroBudget

Table 11 shows the result for our stability metric after applying the previous steps on EzBilling. Our stability metric evaluates the architecture for this system and it gives $100 \%$. We compare a pair of subsequence versions, and we realized the aggregated metric results for all versions are the same.

\begin{tabular}{|l|l|l|l|l|l|l|l|l|l|l|l|}
\hline \multirow{3}{*}{ Ezbilling } & V1 & V2 & V3 & V4 & V5 & V6 & V7 & V8 & V9 & V10 & Stability \\
\cline { 2 - 10 } & 58.19569 & 58.19569 & 58.19569 & 58.19569 & 58.19569 & 58.19569 & 58.19569 & 58.19569 & 58.19569 & 58.19569 & \multirow{2}{*}{$100 \%$} \\
\cline { 2 - 10 } & & 1 & 1 & 1 & 1 & 1 & 1 & 1 & 1 & 1 & \\
\hline
\end{tabular}

Table 11. Stability result for Ezbilling

\section{Conclusion and Future work}

In conclusion, the stability metric that will be used to measure the architecture for a system is designed. There are five steps have to followed in order to calculate the stability for a selected system. These steps are extracting class-level metric using software metrics tool, calculating the average for the class-level metric, calculating the aggregated metric, do comparison between the system versions and measuring the stability for the selected system. We have demonstrated our stability metric by applying it on five systems with ten versions. In our case study, we started by talking about the benefits of open source systems and we ended by showing the results our stability metric. Table 12 shows the stability results for each one of the selected system. Our stability metrics evaluate the selected systems and based on it is results we realized that the most stabile system is Ezbilling while Eurobudget system is instable. The stability for Catan and SQSim are the same. Without having a large set of versions for a particular system, our stability metric will not be able to work in order to give a useful stability result.

\begin{tabular}{|l|l|l|l|l|l|}
\hline System & EuroBudget & BarCode Generator & Catan & Ezbilling & SQSim \\
\hline Stability & $22 \%$ & $89 \%$ & $67 \%$ & $100 \%$ & $67 \%$ \\
\hline
\end{tabular}

Table 12. Stability result for all systems

\section{References}

[1] Ahmed, M., Rufai, R., AlGhamdi, J., Khan, S. (2004). Measuring architectural stability in object oriented software. Stable Analysis Patterns: A True Problem Understanding with UML, 21

[2] Ebad, S. A., Ahmed, M. A. (2015). Measuring stability of object-oriented software architectures. Software, IET, 9(3), 76-82.

[3] Aversano, L., Molfetta, M., Tortorella, M. (2013). Evaluating architecture stability of software projects. In 2013 20th Working Conference on Reverse Engineering (WCRE) (pp. 417-424). IEEE. (October).

[4] Alshayeb, M., Naji, M., Elish, M. O., Al-Ghamdi, J. (2011). Towards measuring object-oriented class stability. Software, IET, 5(4), 415-424.

[5] Li, W., Etzkorn, L., Davis, C., Talburt, J. (2000). An empirical study of object-oriented system evolution. Information and Software Technology, 42(6), 373-381. 
[6] Abdeen, H., Ducasse, S., Sahraoui, H. (2011). Modularization metrics: Assessing package organization in legacy large objectoriented software. In: Reverse Engineering (WCRE), 2011 18th Working Conference on (p. 394-398). IEEE. (October).

[7] Sethi, K., Cai, Y., Wong, S., Garcia, A., Sant'Anna, C. (2009, September). From retrospect to prospect: Assessing modularity and stability from software architecture. In Software Architecture, 2009 \& European Conference on Software Architecture. WICSA/ECSA 2009. Joint Working IEEE/IFIP Conference on (p. 269-272). IEEE.

[8] Chidamber, S. R., Kemerer, C. F. (1994). A metrics suite for object oriented design. Software Engineering, IEEE Transactions on, $20(6)$ 476-493.

[9] Hassaine, S., Guéhéneuc, Y. G., Hamel, S., Antoniol, G. (2012, March). Advise: Architectural decay in software evolution. In Software Maintenance and Reengineering (CSMR), 2012 16th European Conference on (p 267-276). IEEE.

[10] Jazayeri, M. (2002). On architectural stability and evolution (p. 13-23). Springer Berlin Heidelberg.

[11] Melo, W. (1996, March). Evaluating the impact of object-oriented design on software quality. In: Software Metrics Symposium, 1996., Proceedings of the $3^{\text {rd }}$ International (p. 90-99). IEEE.

[12] Olague, Hector M., et al. "Assessing design instability in iterative (agile) object oriented projects." Journal of Software Maintenance and Evolution: Research and Practice 18.4 (2006): 237-266.

[13] Tonu, S. A., Ashkan, A., Tahvildari, L. (2006, March). Evaluating architectural stability using a metric-based approach. In null (pp. 261-270). IEEE.

[14] Roden, P. L., Virani, S., Etzkorn, L. H., Messimer, S. (2007, September). An empirical study of the relationship of stability metrics and the qmood quality models over software developed using highly iterative or agile software processes. In: Source Code Analysis and Manipulation, 2007. SCAM 2007. Seventh IEEE International Working Conference on (p. 171-179). IEEE.

[15] Yu, L., Ramaswamy, S. (2009). Measuring the evolutionary stability of software systems: case studies of Linux and FreeBSD. Software, IET, 3 (1), 26-36.

[16] Raemaekers, S., van Deursen, A., Visser, J. (2012). Measuring software library stability through historical version analysis. In Software Maintenance (ICSM), $201228^{\text {th }}$ IEEE International Conference on ( $p$ 378-387) (September). IEEE.

[17] Ducasse, S., Gîrba, T., Marinescu, R. (2004). Using History Information to Improve Design Flaws Detection. In: CSMR 2004: $8^{\text {th }}$ European Conference On Software Maintenance and Reengineering.

[18] Bansiya, J. (2000). Evaluating framework architecture structural stability. ACM Computing Surveys (CSUR), 32 (1es), 18.

[19] Alenezi, Mamdouh., Khellah, Fakhry. (2015). Evolution Impact on Architecture Stability in Open-Source Projects. International Journal of Cloud Applications and Computing (IJCAC) 5.4. 24-35.

[20] Martin, R. C. (2003). Agile Software Development: Principles, Patterns, and Practices. Prentice Hall PTR, Upper Saddle River, NJ, USA.

[21] Gomaa, H. (2011). Software modeling and design: UML, use cases, patterns, and software.

[22] Architectures. Cambridge University Press.ISO/IEC/IEEE. Systems and software engineering architecture description. ISO/ IEC/IEEE 42010:2011(E) (Revision of ISO/IEC 42010:2007 and IEEE Std 1471-2000) 1-46, 12011

[23] Chidamber, S., Kemerer, C. (1994). A metrics suite for object oriented design. IEEE Transactions on Software Engineering; 20 (6) 476-493.

[24] Zuse, H. (1997). A Framework of Software Measurement. Walter de Gruyter \& Co.: New York NY; 46, 126-128, 130-151,158239, 399-402.

[25] Malhotra, Ruchika. (2015). Empirical Research in Software Engineering: Concepts, Analysis, and Applications. CRC Press.

[26] Quah, Tong-Seng., Mie Mie Thet Thwin. (2003). Application of neural networks for software quality prediction using objectoriented metrics. Software Maintenance. ICSM 2003. In: Proceedings. International Conference on. IEEE.

[27] Ding, Wei. (2014). How do open source communities document software architecture: An exploratory survey. In: Engineering of Complex Computer Systems (ICECCS), 2014 19th International Conference on. IEEE.

[28] Bakar, Normi Sham Awang Abu. (2016).The Analysis of Object-Oriented Metrics in C++ Programs. Lecture Notes on

$46 \quad$ Journal of Information Technology Review Volume 9 Number 2 May 2018 
Software Engineering 4.1. 48.

[29] https://sourceforge.net/

[30] http://www.virtualmachinery.com/jhawkprod.htm 